Any criticism of the foregoing views must be confined to the main points. In the matter of theories it may be said with every deference that "safety is not in numbers." Three theories should not be required to explain one plain fact. If differing, they suffer mutual defeat; if similar, then their agreement should be more absolute than it is in this case, to fit a quantity so strictly constant as the "triangle."

This onstanoy is the second consideration of a general order which is to the point. Grocco's triangle was originally introduced to us in terms of angles and of millimetres, its height alone undergoing variation; although the pleural effusions vary extensively in bulk. Subsequently, however, when I discovered in 1905 that it can also be produced by collections below the diaphragm, and in particular by ascites, I was able to point out that the base of the triangle was apt to be broader in the abdominal eases. In short, it may be said of all three theories that they agree neither among themselves nor with the mathematical feature of the phenomenon they ought to explain.

Passing now to details, a principle of fundamental importance to clinicians in their study of intra-pleural events is the fact that a lung laterally compressed by fluid retreats into the spinal groove by virtue of the posterior position of the pulmonary root above and of the attachment below of the posterior edge of the lung by means of the ligamentum latum pulmonis. This recession and this attachment have always seemed to me to govern in effusion the persistence of the spinal and vertebral resonance for some distance below the upper level of the fluid, and the production of the S-shaped outline of dulness of Damoiseau, and they must not be lost sight of in the present connexion. In upward compressions from abdominal lifting of the diaphragm the same principle should hold good; and it is not easy to realise how the posterior pulmonary border could possibly vacate its groove.

Another principle is that whatever may happen to the anterior pulmonary border by way of collapse or of enlargement or of encroachment into the other half of the thorax the pulmonary root and a fortiori the posterior border are incapable of any migration across the vertebral column. This is precluded by the attachments of the centrum tendineum to the spine on the one hand, and to the inferior vena cava on the other. The posterior and vertical axis of rotation of the heart which passes through the foramen pro vena cava from the liver up to the superior vena cava and the innominate artery may be stretched in its upper part, and lower down it may sway slightly with the centrum tendineum and with the liver under the stress of powerful pressures, as in Professor Elliot Smith's case. But this lateral displacement can amount only to very little, and the close connexion of the spinal column with the pericardium and the posterior mediastinum, kept up through the vena azygos, raises an impassable barrier between the two spinal grooves, precluding any transference of viscera from the one into the other.

There is, moreover, a new clinical fact which seems to leave little standing ground for any theory dependent upon alleged oscillations of fluids or of viscera across the middle line. I have more recently realised that a Grocco's triangle can be percussed out on both sides of the spine in bilateral pleural effusions, as well as in ascites. This is easily demonstrated by the skilful use of Sansom's pleximeter. The significance of this fact from the point of mechanism is obvious. The two triangles could not be produced by reciprocal encroachment by bulgings, but they can be explained as the result of reciprocal percussion waves. A pleximetric conduction would also seem to be the only explanation available for the telling fact that the two triangles are almost always of uneven height as the two effusions are rarely equal.

The conclusions may be briefly stated:-

1. Grocco's paravertebral thoracic triangle of dulness, due to fluid free in the pleura, is rectilinear not curved; and in other respects also it agrees with the description of it given by Grocco.

2. It is also produced by fluid collections in the abdomen; its base is then broader than in pleural cases.

3. In the latter neither its width nor its shape vary appreciably, but only its height; this rises exactly to the level reached by the effusion itself.

4. In bilateral cases of pleural effusion a Grocco's triangle can be made out on both sides, in spite of the dulnesses due to the two effusions. As the effusions are seldom quite equal two unequal Grocco's triangles are the rule.
5. In ascites and analogous abdominal cases the, rule is for the two triangles to be equal; they therefore make up together a low but wide equilateral triangle bisected by the spine

6. The simultaneous occurrence of a right and of a left Grocco's triangle is incompatible with the alleged causation by pleural bulging across the vertebral column.

7. The same reasoning applies to the theory that a pendulum displacement of the intra-thoracic viscera across the middle line might explain the dulness.

8. Anatomical probabilities are against any possible shifting of a collapsed pulmonary base away from its own vertebral groove.

9. The assumption that a Grocco's triangle is produced directly by any fluid or other non-resonant substance immediately underlying it is negatived by the circumstance that the general outline of the triangle is always "geometrically" the same and is not contingent upon the innumerable variations in size and in shape which belong to fluid collections or to solidifications.

10. This is an argument in favour of the causation suggested under the name of the pleximetric spine theory.

11. The same view is supported by the results of the oruoial test and counter test, which also afford proof of the genuineness of this clinical sign and of its helpfulness in the diagnosis of pleural and abdominal conditions.

Upper Brook-street, $W$.

\section{PANCREATIC GLYCOSURIA TEN YEARS AFTER TYPHOID FEVER.}

\section{BY P. J. OAMMIDGE, M.D. LoND.}

ON Sept. 8th, 1908, I was consulted by an American gentleman, who came to me with a letter of introduction from Dr. J. B. Murphy of Chicago. In his letter Dr. Murphy stated that the patient was suffering from diabetes and had been sent to me with the object of determining the condition of his pancreas and clearing up the etiology of the disease. He also informed me that there was a distinct history of cholecystitic infection.

The patient was a well-built, healthy looking man, 51 years of age. He had had no serious illness, except an attack of typhoid fever in 1898. There was no history of syphilis or gastro-intestinal disturbances, nor had he had any symptoms pointing to the presence of gall-stones. Although he smoked a good deal he took little or no alcohol. He had never suffered from thirst or polyphagia. The quantity of urine excreted had not increased. So far as he knew he had not lost flesh, nor had he noticed any diminution of strength. 3 per cent. of sugar had been found in his urine in the course of a routine examination at an American health resort in January, 1908, but on an anti-diabetic diet this had been reduced to about half.

A physical examination revealed nothing abnormal. The patient's tongue was not red or glazed, and his gums and mouth appeared normal. His skin was moist. His heart was not enlarged, the heart sounds were natural, and his arteries were not thickened. His abdomen and body generally were well covered with fat. No abdominal tumour or swelling could be discovered on palpation. The liver dulness was normal and the stomach did not appear to be dilated. Analysis of a specimen of the mixed night and morning urine gave the following results :-

Reaction, acid; specific gravity, 1023 ; albumin, about 1: 5000 ; sugar, Fehling's solution, reduced at once, phenylhydrazin, crowds of typical phenylglucosazone crystals, insoluble in 33 per cent, sulphurie acid in five minutes. Quantitatively, copper reduction (Bang's method), $2 \cdot 3$ per cent. Polariscope, $+2 \cdot 2$ per cent. Fermentation (Lohenstein's saccharimeter), 1.9 per cent.); acetone, nil ; aceto-acetic acid, nil ; indican, a fairly well-marked reaction; bile, $n i l$ : urobilin, a pathological excess: blood, nil ; urea, 2.42 per cent.; chlorides, 0.8 per cent. phosphates, 0.13 per" cent.; preformed to conj. sulphates, $8: 1$. "Critical solution point" (phenol), raised $10^{\circ} \mathrm{C}$ Hricroscopically, man small calcium oxalate crystals, a few squamous and transitional epithelial cells, a few leucocytes, no casts. "Pancreatic" reaction, many typical fine crystals, soluble in 33 per cent. sulphuric acid in $5-10$ seconds: melting point, after re-crystallisation, $160^{\circ} \mathrm{C}$

The urine, therefore, contained a fair amount of a dextrorotatory fermentable sugar, with traces of a non-fermentable variety. The results of the "pancreatic" reaction suggested that the glycosuria was probably associated with disease of the pancreas, and the presence of many small calcium 
oxalate crystals in the centrifugalised deposit tended to confirm this conclusion. The pathological excess of urobilin pointed to there being a catarrhal condition of the biliary passages, which probably extended to the pancreatic ducts. The abnormal reaction for indican and the disturbed relation of the preformed to the conjugated sulphates suggested that both the pancreatic disease and cholangitis were connected with a catarrhal condition of the upper part of the intestinal tract. The absence of any trace of acetone or aceto-acetic acid showed that there were not the profound tissue changes met with in severe diabetes and rendered it probable that the pancreatic disease was of a slowly advancing type, such as, in my experience, is the common result of cirrhosis of the pancreas secondary to infection of the ducts. Although the specimen contained traces of albumin, the fairly normal critical solution point, the normal percentages of urea and inorganic salts, and the absence of casts or other evidence of renal mischief rendered it unlikely that there was any serious disease of the kidneys, while the presence of leucocytes and transitional epithelium suggested that it was not improbabiy of bladder origin.

The bowels were stated to be opened regularly every day, and there was neither diarrhœea nor constipation. A sample of the frecs submitted for examination gave the following results :-

Appearance, dark brown, formed, solid mass; reaction, amphoteric stercobilin, a well-marked reaction; occult blood, nil. Microscopically, a little vegetable tissue, many partly digested muscle fibres, some fatty acid and soap crystals, no fat globules;

\begin{tabular}{|c|c|c|c|c|c|c|}
\hline Organic matter... & ... & $86.7 \%$ & & & & $85-897$ \\
\hline Total fat... ... ... & ... & $16.6 \%$ & $"$ & $"$ & $"$ & $15-25 \%$ \\
\hline Unsaponified fat... & ... & $8 \cdot 6 \%$ & ", & $"$ & $"$ & $10-15 \%$ \\
\hline Saponified fat $\quad \ldots$ & $\ldots$ & $8 \cdot 0 \%$ & $"$ & ", & $"$ & $10-15 \%$ \\
\hline Organie matter not fat & $\ldots$ & $70 \cdot 1 \%$ & $" 9$ & " & ", & $60-75 \%$ \\
\hline Inorganic ash $\quad \ldots \quad \ldots$ & $\ldots$ & $13 \cdot 8 \%$ & ," & $"$ & , & $10-15 \%$ \\
\hline
\end{tabular}

The only striking variation from the normal was the large amount of partly digested muscle fibre found microscopically, but this was probably to be explained by the highly nitrogenous diet which the patient was taking. There was no marked excess of unabsorbed fat, nor was the relation between the saponified and unsaponified fats disturbed in the way that one might expect in serious disease of the pancreas. This, however, I have found in a considerable number of cases of glycosuria of undoubted pancreatic origin in which I have had the opportunity of examining the stools, and I have come to the conclusion that any marked interference with fat digestion is of serious prognostic significance and indicates that the patient is in the last stages of the disease. The absence of any trace of occult blood was against there being any malignant growth in the course of the gastrointestinal tract, to which the pancreatic disease might be secondary.

As I have pointed out elsewhere, ${ }^{1}$ I have been led to divide cases of pancreatic diabetes into three classes, according to the probable origin of the pancreatic affection:-(1) Those in which the morbid influence has reached the gland by way of the ducts; (2) those secondary to blood diseases or circulatory disturbances; and (3) those in which the diabetes has been induced by destruction of the pancreas by malignant growths. There was no evidence that the case under consideration fell under either the second or third head, while the urinary analysis suggested that it was associated with an infection of the pancreatic ducts and biliary passages.

Bearing in mind the history of typhoid fever, and the now well-recognised tendency of the typhoid bacillus to linger in the gall-bladder and bile-ducts, in some cases, long after the patient has quite recovered from the disease, it seemed to me possible that I had to do with a case of typhoidal pancreatitis going on to glycosuria, and that a bacteriological analysis of the fæces might throw further light on the condition. A portion of the fæcal material was taken with a sterile knife from the centre of the mass sent for examination, emulsified in sterile normal saline solution, plated on Drigalski and Conradi's medium, and incubated at $37^{\circ} \mathrm{C}$. On being examined 24 hours later one of the six plates that had been inoculated showed two small, blue, finely granular colonies, with raised centres and filmy edges, suggesting bacillus typhosus. Sub-cultures were made from these on to agaragar slopes and incubated at $37^{\circ} \mathrm{C}$. for 24 hours; they then

1 Surgery, Gynecology, and Obstetrics, January, 1908, p. 22. showed a thin, bluish-white, translucent film, that had not spread far from the needle track. Hanging-drop preparations showed actively motile rod-shaped bacilli. The bacilli did not stain by Gram's method, and cover-glass preparations stained by Van Ermengen's method showed that they were richly flagellate. Glucose-gelatine shake cultures showed no gas formation in 48 hours. Lactose litmus broth was not rendered acid and showed no gas formation. Litmus-milk cultures were not coagulated, nor was there any acid formation in 48 hours. Neutral red broth became turbid, but there was no film formation or colour change. Peptone salt cultures incubated at $37^{\circ} \mathrm{C}$. for 48 hours gave no indol reaction. An emulsion of the bacilli in normal saline solution, prepared from a 24 hours agar culture, gave a similar reaction with a typhoid serum to a laboratory cuiture of bacillus typhosus. The fæces, therefore, contained a small number of bacilli having the appearance and characters of bacillus typhosus; a result which tended to confirm my surmise as to the probable origin of the disease.

Pancreatitis is not a common sequel of typhoid fever, and as yet there has been no reported instance in which glycosuria has developed as a result of typhoidal infection of the pancreas. The interval of ten years that had elapsed in this case between the original infection and the discovery of sugar in the urine, at first sight, made it a little difficult to believe that there could be any etiological relation between the two, but as the bacteriological examination of the stools showed that typhoid bacilli were still present, and there was evidence pointing to a focus of infection in the biliary passages and pancreatic ducts, it was not too much to assume that such a relation existed, and that the glycosuria was the direct outcome of slowly advancing changes in the pancreas set up by the persistent infection. Contrary to what has been generally taught, I have found that pancreatic diabetes is usually a mild and slowly advancing disease, often unattended by any marked symptoms for many years, ${ }^{2}$ so that the absence of any physical signs and the latent character of the disease in this case did not surprise me but rather tended. to confirm my view as to the cause of the glycosuria.

In the treatment of pancreatic diabetes it is essential to first determine the cause of the condition of the pancreas and endeavour so far as possible to control it. As it was highly probable that in this case the primary etiological factor was an infection of the pancreatic ducts and biliary system with typhoid bacilli advantage was taken of the results of the experiments by S. J. Crowe ${ }^{3}$ in which it was shown that hexamethylenetetramine (urotropine) given by the mouth to dogs is excreted in the bile and pancreatic juice in quantity and speedily sterilises the ducts that have been artificially infected with typhoid bacilli. Ten grains of this drug, with ten grains of sodium salicylate, which is also excreted in the bile and pancreatic juice, were therefore ordered to be taken three times a day in two ounces of water for three weeks, when it was suggested that the fæces and urine should be again examined. The second indication for treatment is to discover the patient's tolerance and powers of digestion for the various forms of sugar (particularly levulose and dextrose), starch, fat, and proteid, so that a diet may be arranged which, while it throws a minimum amount of work upon the pancreas and combats the glycosuria, may yet supply a sufficient quantity of nourishment to keep the patient in good condition. The only really satisfactory way of arriving at such a diet is to have the patient under observation in a nursing home or hospital for a short time so that the food may be accurately prescribed in weighed quantities and the effects of variations, with and without the addition of preparations of pancreas, can be determined by regular quantitative analyses of the urine and fæces. Such a procedure also has the advantage of educating the patient to a new mode of life and placing him in a much better position to take care of himself subsequently.

As this patient was obliged to return to America immediately after I had seen him I was only able to give him general directions as to his diet and mode of life, based upon my experience of similar cases, and suggest that he should consult his medical adviser in Chicago and get him to work out a diet chart suitable to his needs.

Beaumont-street, W.

2 The Pancreas, its Surgery and Pathology, by Mayo Robson and

Cammidge, pp. 269 and 438. 